\title{
Left Ventricular Geometry and Replacement Fibrosis Detected by cMRI Are Associated with Major Adverse Cardiovascular Events in Nonischemic Dilated Cardiomyopathy
}

\author{
Bianca Olivia Cojan-Minzat ${ }^{1,2}$, Alexandru Zlibut ${ }^{1}$, Ioana Danuta Muresan ${ }^{1}$, Carmen Cionca ${ }^{3}$, \\ Dalma Horvat ${ }^{1}$ (D), Eva Kiss ${ }^{1}$, Radu Revnic ${ }^{2}$, Mira Florea ${ }^{2}$, Razvan Ciortea ${ }^{1,4}$ and \\ Lucia Agoston-Coldea 1,3,5,* \\ 1 Department of Internal Medicine, Iuliu Hatieganu University of Medicine and Pharmacy, \\ 400006 Cluj-Napoca, Romania; cojanminzat.bianca@yahoo.com (B.O.C.-M.); alex.zlibut@yahoo.com (A.Z.); \\ ioanamuresandanuta@yahoo.com (I.D.M.); hdalma92@yahoo.com (D.H.); kiss.eva24@yahoo.com (E.K.); \\ r_ciortea@yahoo.com (R.C.) \\ 2 Department of Family Medicine, Iuliu Hatieganu University of Medicine and Pharmacy, \\ 400001 Cluj-Napoca, Romania; radu_revnic@yahoo.com (R.R.); mira_florea@yahoo.com (M.F.) \\ 3 Department of Radiology, Affidea Hiperdia Diagnostic Imaging Center, 400015 Cluj-Napoca, Romania; \\ carmen.cionca@yahoo.com \\ 4 Department of Obstetrics and Gynecology, Emergency County Hospital, 400124 Cluj-Napoca, Romania \\ 5 2nd Department of Internal Medicine, Emergency County Hospital, 400006 Cluj-Napoca, Romania \\ * Correspondence: luciacoldea@yahoo.com; Tel.: +402-6459-1942; Fax: +402-6459-9817
}

Received: 7 May 2020; Accepted: 22 June 2020; Published: 25 June 2020

\begin{abstract}
To investigate the relationship between left ventricular (LV) long-axis strain (LAS) and LV sphericity index (LVSI) and outcomes in patients with nonischemic dilated cardiomyopathy (NIDCM) and myocardial replacement fibrosis confirmed by late gadolinium enhancement (LGE) using cardiac magnetic resonance imaging (cMRI), we conducted a prospective study on 178 patients ( $48 \pm 14.4$ years; $25.2 \%$ women) with first NIDCM diagnosis. The evaluation protocol included ECG monitoring, echocardiography and cMRI. LAS and LVSI were cMRI-determined. Major adverse cardiovascular events (MACEs) were defined as a composite outcome including heart failure (HF), ventricular arrhythmias (VAs) and sudden cardiac death (SCD). After a median follow-up of 17 months, patients with LGE+ had increased risk of MACEs. Kaplan-Meier curves showed significantly higher rate of MACEs in patients with LGE $+(p<0.001)$, increased LVSI $(p<0.01)$ and decreased LAS $(p<0.001)$. In Cox analysis, LAS (HR $=1.32,95 \% \mathrm{CI}(1.54-9.14), p=0.001)$, LVSI [HR $=1.17,95 \% \mathrm{CI}$ (1.45-7.19), $p<0.01]$ and LGE+ (HR $=1.77,95 \%$ CI $(2.79-12.51), p<0.0001)$ were independent predictors for MACEs. In a 4-point risk scoring system based on LV ejection fraction (LVEF) $<30 \%$, LGE+, LAS $>-7.8 \%$ and LVSI $>0.48 \%$, patients with 3 and 4 points had a significantly higher risk for MACEs. LAS and LVSI are independent predictors of MACEs and provide incremental value beyond LVEF and LGE+ in patients with NIDCM and myocardial fibrosis.
\end{abstract}

Keywords: nonischemic dilated cardiomyopathy; cardiac magnetic resonance imaging; late gadolinium enhancement; long axis strain; left ventricle sphericity index; major adverse cardiovascular events

\section{Introduction}

Nonischemic dilated cardiomyopathy (NIDCM) is the most common primary myocardial disease, being characterized by left ventricular (LV) enlargement and global systolic LV function impairment in the absence of ischemic heart disease (IHD), hypertension or valve disease [1]. Due to its significant 
increased mortality [2] and sudden cardiac death (SCD) risk [3], NIDCM represents an important global healthcare burden. Nowadays, the development of more effective methods of assessing NIDCM severity and the risk of major adverse cardiovascular events (MACEs) remains a topic of great interest for current research.

While current guidelines recommend echocardiography as the first line of investigation in patients with NIDCM [4,5], it cannot evaluate the structural myocardial impairment [6]. Myocardial replacement fibrosis is part of the cardiac remodelling process, being responsible for heart failure (HF), ventricular arrhythmia (VA) and SCD. It is encountered in one-third of NIDCM patients, being detected using cardiac magnetic resonance imaging (cMRI) with late gadolinium enhancement (LGE) [7-10]. T1 mapping imaging is a state-of-the-art cMRI technique that is able to characterize extracellular volume fraction and it has been validated by comparative studies with the histopathological examination in NIDCM [11,12]. LV long-axis strain (LAS) determined by cMRI is an efficient and reliable method for quantifying global LV longitudinal function and it has an important prognostic value in patients with NIDCM [13,14]. Last but not least, cMRI-determined LV sphericity index (LVSI) is a parameter that predicts MACEs in NIDCM and it can be used in the assessment of LGE presence and LGE mass $[15,16]$.

The aim of this study was to investigate the relationship between cardiac remodelling process and MACEs, and if it increases outcome prediction beyond LGE, in patients with NIDCM.

\section{Methods}

\subsection{Study Population}

We conducted a prospective study on 302 consecutive patients with first NIDCM diagnosis, which were examined in the 2th Department of Internal Medicine of the Iuliu Hatieganu University of Medicine and Pharmacy from October 2017 to November 2019. The inclusion criteria were [1]: (1) impaired global LV function with a LV ejection fraction (LVEF) $\leq 45 \%$; (2) LV chamber dilation with an indexed LV end-diastolic volume (LVEDV) $\geq 97 \mathrm{~mL} / \mathrm{m}^{2}$; both cMRI-determined. The exclusion criteria are represented by (1) IHD, other cardiomyophaties, significant valvular and congenital heart disease (CHD, CVD); (2) contraindications to cMRI (incompatible metallic devices, significant chronic renal disease with estimated glomerular filtration rate $<30 \mathrm{~mL} / \mathrm{min} / 1.73 \mathrm{~m}^{2}$, or claustrophobia); (3) refusal to participate in the study (Figure 1). IHD was excluded by coronarography in 72 patients $(41 \%)$, stress imaging studies in 64 patients $(36 \%)$ and the remaining 42 patients $(23 \%)$ had no history of angina, 1 or 0 risk factors for IHD and stress ECG test and computed tomography coronary angiography with Agatston calcium scoring were also negative.

We recorded demographic data including age, gender, height, weight, medical history, cardiovascular symptoms (dyspnoea, syncope, palpitations), and current medication; biomarkers and 12-lead ECG. 24-h Holter monitoring, transthoracic echocardiography and cMRI were performed. The current research was approved by the Ethics Committee of the Iuliu Hatieganu University of Medicine and Pharmacy, Cluj-Napoca-decision number 280/26.07.2018. The study was conducted in accordance with the principles of the Declaration of Helsinki. All patients were informed about the investigation protocol and signed a written consent form. 


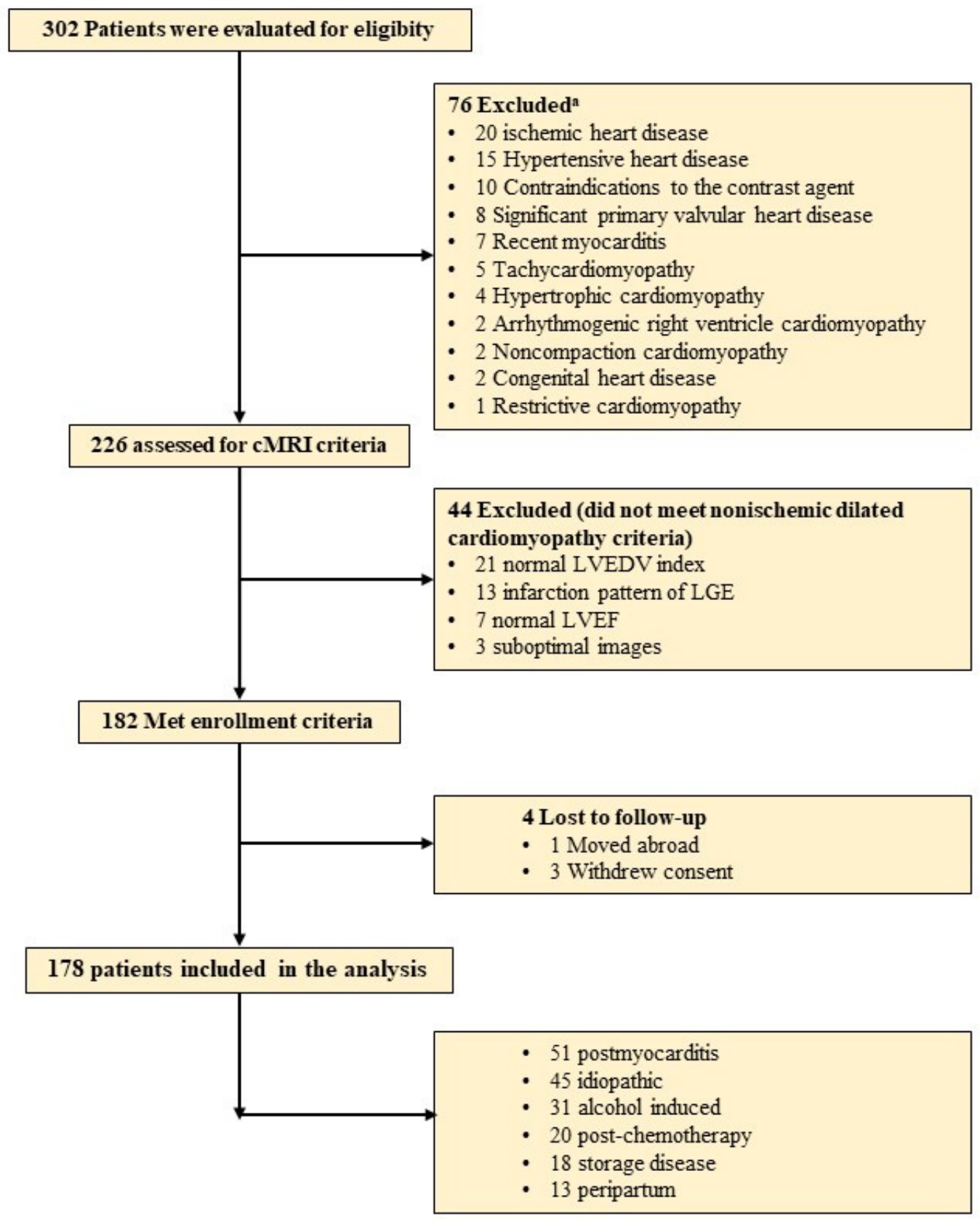

${ }^{a}$ coronary artery disease was defined as $>50 \%$ angiographical stenosis in any epicardial coronary artery. Primary valvular disease was defined as moderate or higher valvular stenosis or regurgitation, with the exception of the functional ones. Functional mitral regurgitation was defined as mitral regurgitation secondary to left ventricular remodeling resulting in failure of leaflet coadaptation, in the setting of normal mitral valve anatomy, on echocardiography and cardiovascular magnetic resonance imaging.

Figure 1. Flow chart detailing the identification of the study cohort. Abbreviations: cMRI, cardiac magnetic resonance imaging; LGE, late gadolinium enhancement; LVEDV, left ventricular end diastolic volume; LVEF, left ventricular ejection fraction.

\section{2. $c M R I$}

All cMRI images were ECG-gated and were acquired during apnoea with a $1.5 \mathrm{~T}$ magnetic resonance (MR) scanner (Magnetom Symphony, Siemens Medical Solutions, Erlanger, Germany). 
A standard scanning protocol that was in accordance with current international guidelines was used [17]. The acquisition of fast imaging employing steady-state free precession (SSFP) sequences was performed to detect ventricular function and mass in the conventional cardiac short-axis and long-axis planes (including two-chamber, three-chamber, and four-chamber), to enclose both ventricles from base to apex. SSFP sequence parameters were as follows: repetition time (TR) $3.6 \mathrm{~ms}$; echo time (TE) $1.8 \mathrm{~ms}$; flip angle $60^{\circ}$; slice thickness $6 \mathrm{~mm}$; field of view $360 \mathrm{~mm}$; image matrix of $192 \times 192$ pixels; voxel size $1.9 \times 1.9 \times 6 \mathrm{~mm} ; 25-40 \mathrm{~ms}$ temporal resolution reconstructed to 25 cardiac phases. LGE imaging was performed to detect focal myocardial scars acquired $10 \mathrm{~min}$ after intravenous administration of $0.2 \mathrm{mmol} / \mathrm{kg}$ gadoxetic acid (Clariscan, GH Healthcare AS, Oslo, Norway) in long- and short axis-views, using a segmented inversion-recovery gradient-echo sequence. LGE imaging sequence parameters were presented by: TR $4.8 \mathrm{~ms}$, TE $1.3 \mathrm{~ms}$, and inversion time 200 to $300 \mathrm{~ms}$. Inversion time was adjusted to optimize nulling of apparently normal myocardium. Brachial blood pressure was monitored during cMRI-SSFP acquisitions.

Image analysis: All images were evaluated by two experienced observers, blinded to all clinical data. LVEDV and LV end-systolic volume (LVESV), LVEF and end-diastolic LV mass (LVM) were measured on short-axis cine-SSFP images. Epicardial and endocardial borders were traced semi-automatically at end-diastole and end-systole using specialized software (Syngo.Via VB20A_HF04, Argus, Siemens Medical Solutions). The maximum left atrium (LA) and right atrium (RA) volumes were measured in all patients from the four-chamber view. All volumes were indexed to body surface area. Tricuspid annular plane systolic excursion (TAPSE) was measured from the mid-four-chamber cardiac view to assess right ventricular (RV) longitudinal motion. LV longitudinal function was assessed by LAS, defined as the difference in mitral annular displacement at end-systole vs. end-diastole, and expressed as a percentage [13]. LVSI was calculated by dividing LVEDV to the volume of a sphere whose LV length $(\mathrm{L})$ is measured at end-diastole: $\mathrm{LVSI}=\mathrm{LVEDV} /\left(\pi / 6 \times(\mathrm{L})^{3}\right)$ [15] (Figure 2).

The presence and distribution of LGE in the LV were assessed from short-axis images, using the 17-segments model, as recommended by the American Heart Association [18], and were quantified using a signal intensity threshold of $>5 \mathrm{SD}$ above a remote reference for normal myocardium. Due to the fact that the LGE quantification with the threshold of 5SD demonstrated the best agreement with visual assessment and best reproducibility among different technique thresholds, we used a threshold of 5SD above the signal intensity of normal myocardium [19,20]. LGE's distribution was characterized as mid-wall, subepicardial, focal or diffuse. The assessment of LGE mass in the LV was automatically quantified from short-axis LGE images using cardiac dedicated software (cvi42, Circle Cardiovascular Imaging Inc., Calgary, CA). The extent of LGE was expressed by gram (g) and also as percentage of LVM. According to the cMRI, the studied population was divided into two groups, namely: patients without LGE (LGE-) and patients with LGE (LGE+).

\subsection{Follow-Up of Clinical Outcomes}

The clinical follow-up was obtained by completing a questionnaire either on hospital visits, telephone house-calls, or both, aiming at delineating the occurrence of the clinical outcomes, which corresponded to the first event occurring in each patient among the following MACEs: death or aborted death from cardiac cause, sustained ventricular tachyarrhythmia (beats with ventricular origin that lasts $>30 \mathrm{~s}$ and has a rate greater than $>100$ beats/min), and HF requiring hospitalization defined accordingly to current international guidelines [4,5]. Hospitalisation due to non-cardiac causes was not counted as event. Survival analysis was performed for the clinical outcomes. The median follow-up was 17 months and maximum follow-up reached 29 months. 

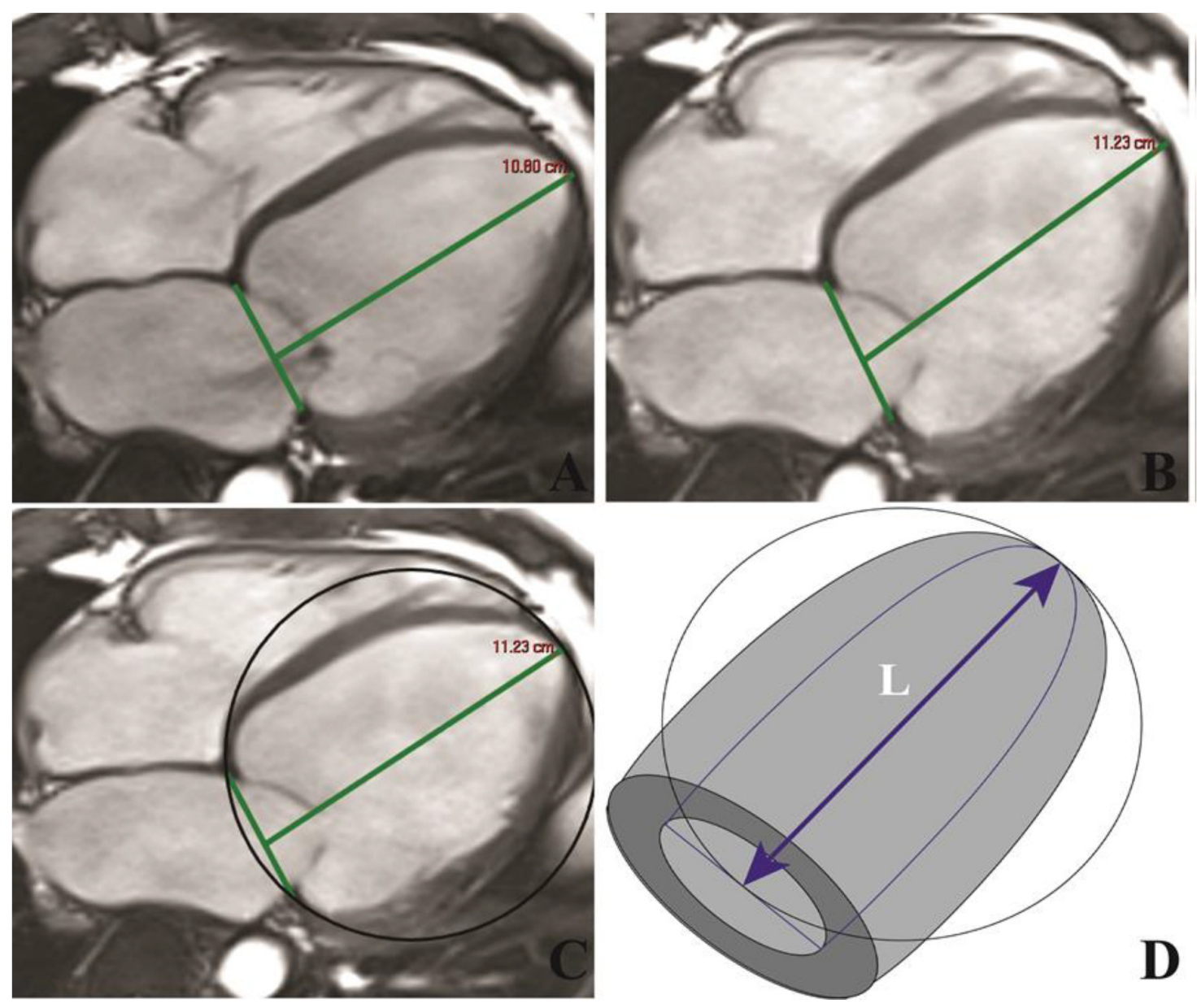

Figure 2. Representative image illustrating the technique for assessment of LAS $(\mathbf{A}, \mathbf{B})$ and LVSI $(C, D)$ in a patient with severe NIDCM in end-diastole and end-systole, respectively. Abbreviations: LAS, left ventricular long axis strain; LVSI, left ventricular sphericity index; NIDCM, non-ischemic dilated cardiomyopathy.

\subsection{Statistical Analysis}

All data were tested for normality using the Kolmogorov-Smirnov test. Data were presented as median, mean \pm standard deviation (SD) or percentage. Baseline characteristics among those with and without LGE patients with clinical outcomes were compared by Chi-square $\chi^{2}$ test or Fischer exact test as appropriate (categorical data) and Wilcoxon signed rank test (continuous data). The hazard ratio (HR) for the prediction of events was calculated using a Cox regression model. For each outcome, we considered all of the significant variables in the univariate analysis and sought the best overall multivariate models for the composite end-point, by stepwise-forward selection, with a probability to enter set at $p<0.05$ and to remove the effect of regression at $p<0.05$. Event-free survival was generated by the Kaplan-Meier method and statistical significance was determined by the log-rank test. Multivariate analysis was performed by constructing a multiple logistic regression model, including the HR $(95 \% \mathrm{CI})$ calculation. Cohen's Kappa inter- and intra-observer coefficient calculation was performed. Retrospective test power calculation and prospective sample size were estimated, with type I and type II variation according to sample size. The statistical analysis was performed using the MedCalc (Version 19.1.7, MedCalc Software, Ostend, Belgium). 


\section{Results}

\subsection{Baseline Characteristics}

A total of 178 patients ( $48 \pm 14.4$ years old, $74.7 \%$ male) met the enrolment criteria (Figure 1 ). They were divided in two groups according to LGE+ and LGE- $(n=64,36 \%$ vs. $n=114,64 \%)$. The baseline characteristics are presented in Table 1.

Table 1. Baseline characteristics of patients in study.

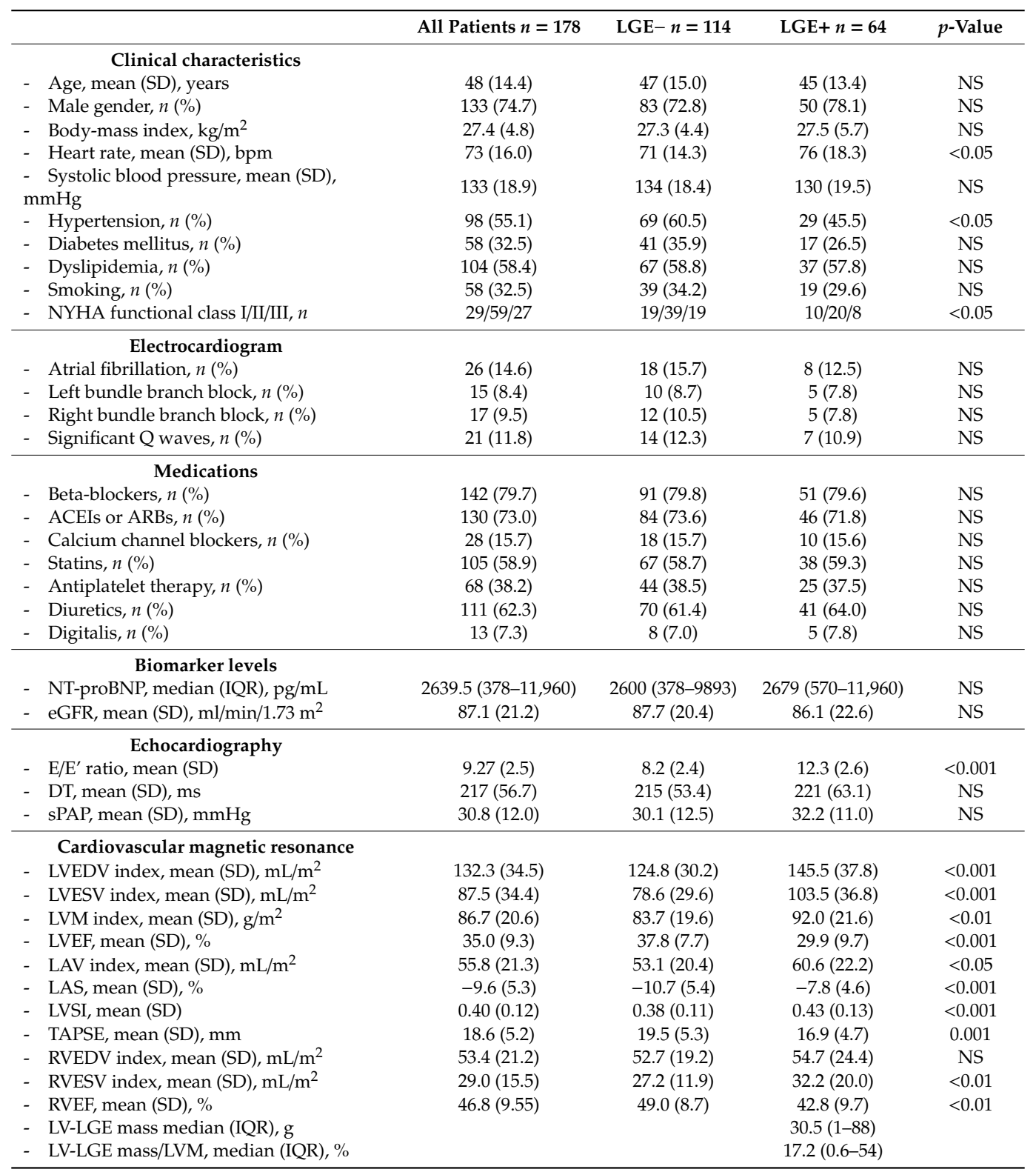

Abbreviations: ACEI, angiotensin converting enzyme inhibitor; ARB, angiotensin receptor blocker; DT, early diastolic filling deceleration time; E, peak mitral flow velocity; E', early diastolic peak myocardial velocity; eGFR, estimated glomerular filtration rate; IQR, interquartile range; LAS, left ventricular longitudinal-axis strain; LAV, left atrial volume; LGE, left ventricular late gadolinium enhancement; LVEDV, left ventricular end-diastolic volume; LVEF, left ventricular ejection fraction; LVESV, left ventricular end-systolic volume; LVM, left ventricular mass; LVSI, left ventricular sphericity index; $n$, number of patients; NT-proBNP, N-terminal pro-Brain Natriuretic Peptide; NYHA, New York Heart Association; RVEDV, right ventricular end-diastolic volume; RVEF, right ventricular ejection fraction; RVESV, right ventricular end-systolic volume; SD, standard deviation; sPAP, systolic pulmonary artery pressure; TAPSE, tricuspid annular plane systolic excursion. Data are reported as mean (standard deviation) or median (IQR) or $n(\%)$. 
At admission, 54 (30.3\%) presented with dyspnoea, 28 (15.7\%) with palpitations and $18(10.1 \%)$ had history of syncope. LGE+ patients had significantly increased LV end-diastolic filling pressures $\left(\mathrm{E} / \mathrm{E}^{\prime}\right.$ ratio; $\left.p<0.001\right)$ and many more of these had LVEF $<30 \%(n=46,71.8 \%)$. The LGE + group presented with increased LVM index $\geq 92 \mathrm{~g} / \mathrm{m}^{2}(p=0.01)$, LVEDV $\geq 145.5 \mathrm{~mL} / \mathrm{m}^{2}(p<0.001)$, LVSI $\geq 0.43$ $(p<0.001)$ and reduced LAS $<-7.8 \%(p<0.001)$.

\subsection{Reproducibility of cMRI Measurements}

cMRI measurements were repeatedly performed on the same set of images, acquired from all patients in the study group. Regarding LVEF, LAS, LVSI and the assessment of LGE+, the intra- and inter-observer reproducibility were excellent. The inter-observer kappa coefficients of agreement were 0.91 for LVEF, 0.97 for LAS, 0.93 for LVSI and 0.88 for LGE+, while the intra-observer kappa coefficients of agreement were 0.98 for LVEF, 0.98 for LAS, 0.92 for LVSI and 0.90 for LGE+ (Table 2).

Table 2. Reproducibility inter and intra-reader agreement of cMRI measurements.

\begin{tabular}{cccc}
\hline Parameters & Coefficient Kappa & 95\% Confidence Interval & Standard Error \\
\hline LVEF & 0.91 & Inter-observer \\
LAS & 0.97 & 0.872 to 0.941 & 0.026 \\
LGE & 0.88 & 0.909 to 0.989 & 0.012 \\
LVSI & 0.93 & 0.771 to 0.939 & 0.066 \\
LVEF & 0.956 to 0.952 & 0.029 \\
LAS & 0.98 & Intra-observer \\
LGE & 0.90 & 0.977 to 0.992 & 0.009 \\
LVSI & 0.92 & 0.967 to 0.991 & 0.004 \\
\end{tabular}

Abbreviations: LAS, left ventricular longitudinal-axis strain; LGE, left ventricular late gadolinium enhancement; LVEF, left ventricular ejection fraction; LVSI, left ventricular sphericity index.

\subsection{Survival Analysis}

Initial cMRI evaluation was performed. During a median follow-up of 17 months (IQR 1 to 29 months), 31 patients $(17.4 \%)$ experienced MACEs: VA $(n=14)$, HF requiring hospitalization $(n=11)$, and SCD $(n=6)$. The patients with VA were majority males, had increased LVESV (mean 107,533 mL, $p<0.001)$, decreased LVEF $(28,429 \%, p<0.0001)$, increased LGE mass $(24,5 \mathrm{~g}, p<0.0001)$ and LVSI $(0.46, p<0.001)$. Of them, ten received ICD therapy and 4 were ablated due to implantable cardioverter defibrillator therapy refusal, the last had a mean LVEF around 30\%, two of them had NIDCM post-myocarditis and two were idiopathic NIDCM. The incidence of MACEs was significantly higher in the LGE+ group vs. the LGE- group ( $n=21,67.7 \%$ vs. $n=10,33.4 \%$ ). The Kaplan-Meier curves for event-free survival showed a significantly higher rate of MACEs in patients with LGE+ $(\mathrm{HR}=4.02$; 95\%CI (1.91-8.45), $p<0.001)$, high LVSI (HR = 3.23; 95\%CI (1.59-6.53), $p<0.01)$ and decreased LAS (HR $=3.94 ; 95 \% \mathrm{CI}(1.93-8.03), p<0.001)$ (Figure 3 ).

\subsection{Univariate and Multivariate Cox Analysis}

Among the evaluated parameters, in univariate analysis and multivariate Cox regression analysis, only four remained independent predictors for MACEs, namely LGE $+(\mathrm{HR}=1.77,95 \% \mathrm{CI}$ (2.79 to 12.51), $p<0.0001$ ), reduced LAS (HR $=1.32,95 \% \mathrm{CI}(1.54$ to 9.14), $p<0.001)$ and increased LVSI (HR $=1.17$, $95 \% \mathrm{CI}(1.45$ to 7.19$), p<0.001)$ and LGE mass $(\mathrm{HR}=1.43,95 \% \mathrm{CI}(1.01-6.12), p<0.001)$ (Table 3$)$. 

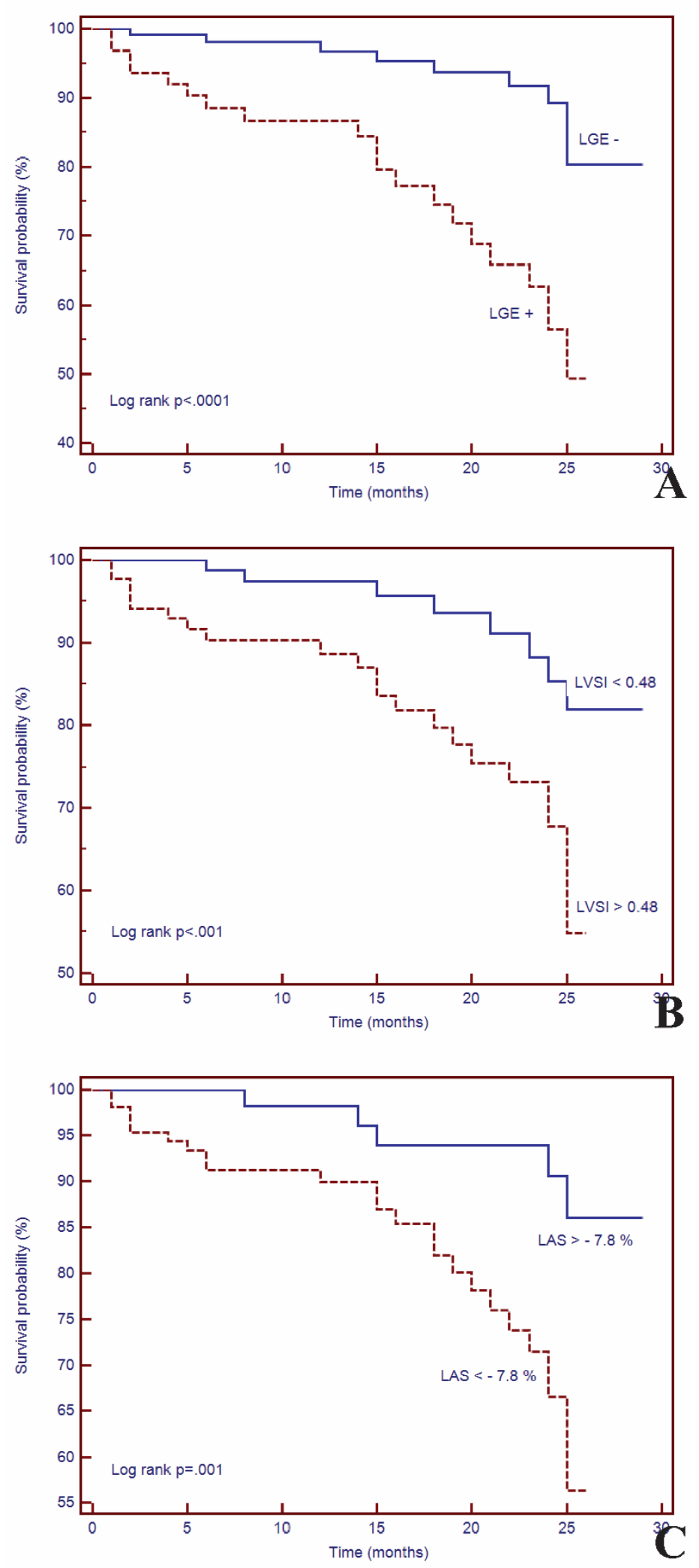

Figure 3. Kaplan-Meier curves for event-free survival for (A) LGE+, (B) LAS, (C) LVSI. Abbreviations: LAS, long axis strain; LGE, left ventricular late gadolinium enhancement; LVSI, left ventricular sphericity index. 
Table 3. Univariable and multivariable Cox analysis testing between studied parameters and MACEs.

\begin{tabular}{|c|c|c|c|c|c|c|}
\hline & \multirow{2}{*}{ No Events $n=147$} & \multirow{2}{*}{ Events $n=31$} & \multicolumn{2}{|c|}{ Univariable Analysis } & \multicolumn{2}{|c|}{ Multivariable Analysis } \\
\hline & & & Unadjusted HR (95\% CI) & $p$ Value & Adjusted HR (95\% CI) & $p$ Value \\
\hline Age, years & $48(13.8)$ & $48(17.5)$ & $1.00(0.98-1.03)$ & NS & & \\
\hline Male gender, $n, \%$ & $111(75.5)$ & $22(37.9)$ & $1.14(0.53-2.48)$ & NS & & \\
\hline $\begin{array}{l}\text { Body-mass index } \\
\mathrm{kg} / \mathrm{m}^{2}\end{array}$ & $27.7(4.8)$ & $25.8(4.5)$ & $0.94(0.87-1.01)$ & NS & & \\
\hline $\begin{array}{l}\text { Systolic blood } \\
\text { pressure }\end{array}$ & $134(19.2)$ & $130(17.4)$ & $0.99(0.97-1.01)$ & NS & & \\
\hline NT-proBNP, pg/mL & $2564(378-11960)$ & $2834(834-9892)$ & $1.00(0.99-1.01)$ & NS & & \\
\hline $\mathrm{eGFR}, \mathrm{ml} / \mathrm{min} / 1.73 \mathrm{~m}^{2}$ & $86.3(20.1)$ & $91.0(25.9)$ & $1.01(0.97-1.03)$ & NS & & \\
\hline LVEDV index, $\mathrm{mL} / \mathrm{m}^{2}$ & $131.4(35.6)$ & $136.6(32.7)$ & $1.01(0.99-1.01)$ & NS & & \\
\hline LVESV index, $\mathrm{mL} / \mathrm{m}^{2}$ & $86.4(34.5)$ & $93.1(33.8)$ & $1.05(0.98-1.07)$ & NS & & \\
\hline LVM index, $\mathrm{g} / \mathrm{m}^{2}$ & $87.0(20.7)$ & $85.2(20.8)$ & $0.99(0.97-1.01)$ & NS & & \\
\hline LVEF, \% & $35.5(9.2)$ & $32.4(9.4)$ & $0.97(0.93-1.01)$ & NS & & \\
\hline LAV index, $\mathrm{mL} / \mathrm{m}^{2}$ & $54.7(21.7)$ & $61.3(18.6)$ & $1.01(1.00-1.03)$ & NS & & \\
\hline LGE + & $43(29.2)$ & $21(67.7)$ & $4.03(1.90-8.52)$ & $<0.0001$ & $1.77(2.79-12.51)$ & $<0.0001$ \\
\hline LGE mass, $\mathrm{g}$ & $11.3(10.6)$ & $28.8(19.3)$ & $1.23(1.90-4.52)$ & $<0.0001$ & $1.43(1.01-6.12)$ & $<0.001$ \\
\hline LAS, $\%$ & $-10.0(5.6)$ & $-7.8(3.6)$ & $1.19(1.01-2.18)$ & $<0.001$ & $1.32(1.54-9.14)$ & 0.001 \\
\hline LVSI, \% & $0.38(0.11)$ & $0.48(0.13)$ & $2.13(1.05-8.11)$ & $<0.001$ & $1.17(1.14-7.19)$ & $<0.01$ \\
\hline $\mathrm{E} / \mathrm{E}^{\prime}$ ratio & $9.1(2.3)$ & $15.7(4.8)$ & $1.08(0.95-1.22)$ & $<0.05$ & $1.02(0.92-1.01)$ & NS \\
\hline TAPSE, $\mathrm{mm}$ & $18.8(5.2)$ & $17.4(5.4)$ & $0.77(0.70-0.84)$ & NS & & \\
\hline RVEDV index, $\mathrm{mL} / \mathrm{m}^{2}$ & $53.3(19.7)$ & $53.8(27.6)$ & $1.00(0.98-1.02)$ & NS & & \\
\hline RVESV index, $\mathrm{mL} / \mathrm{m}^{2}$ & $28.2(13.5)$ & $32.4(13.9)$ & $1.02(1.00-1.04)$ & NS & & \\
\hline RVEF, \% & $47.5(9.3)$ & $43.4(10.2)$ & $0.84(0.79-0.88)$ & NS & & \\
\hline
\end{tabular}

Abbreviations: E, peak mitral flow velocity; E', early diastolic peak myocardial velocity; eGFR, estimated glomerular filtration rate; IQR, interquartile range; LAS, left ventricular longitudinal-axis strain; LAV, left atrial volume; LGE, left ventricular late gadolinium enhancement: LVEDV, left ventricular end-diastolic volume; LVEF, left ventricular ejection fraction; LVESV, left ventricular end-systolic volume; LVM, left ventricular mass; LVSI, left ventricular spherical index; $n$, number of patients; NT-proBNP, N-terminal pro-Brain Natriuretic Peptide; RVEDV, right ventricular end-diastolic volume; RVEF, right ventricular ejection fraction; RVESV, right ventricular end-systolic volume; TAPSE, tricuspid annular plane systolic excursion. 


\subsection{Incremental Predictive Value of cMRI-Based LV Geometry and Strain for Outcomes}

Sequential Cox proportional-hazards models yielded significantly increased predictive power the combined outcome of MACEs when both LVSI and LAS were used in addition to LVEF and LGE+ (Chi-square $=24.52, p<0.0001)$ (Figure 4). However, LAS did not provide incremental predictive power when used alone, in addition to LVEF and LGE+.

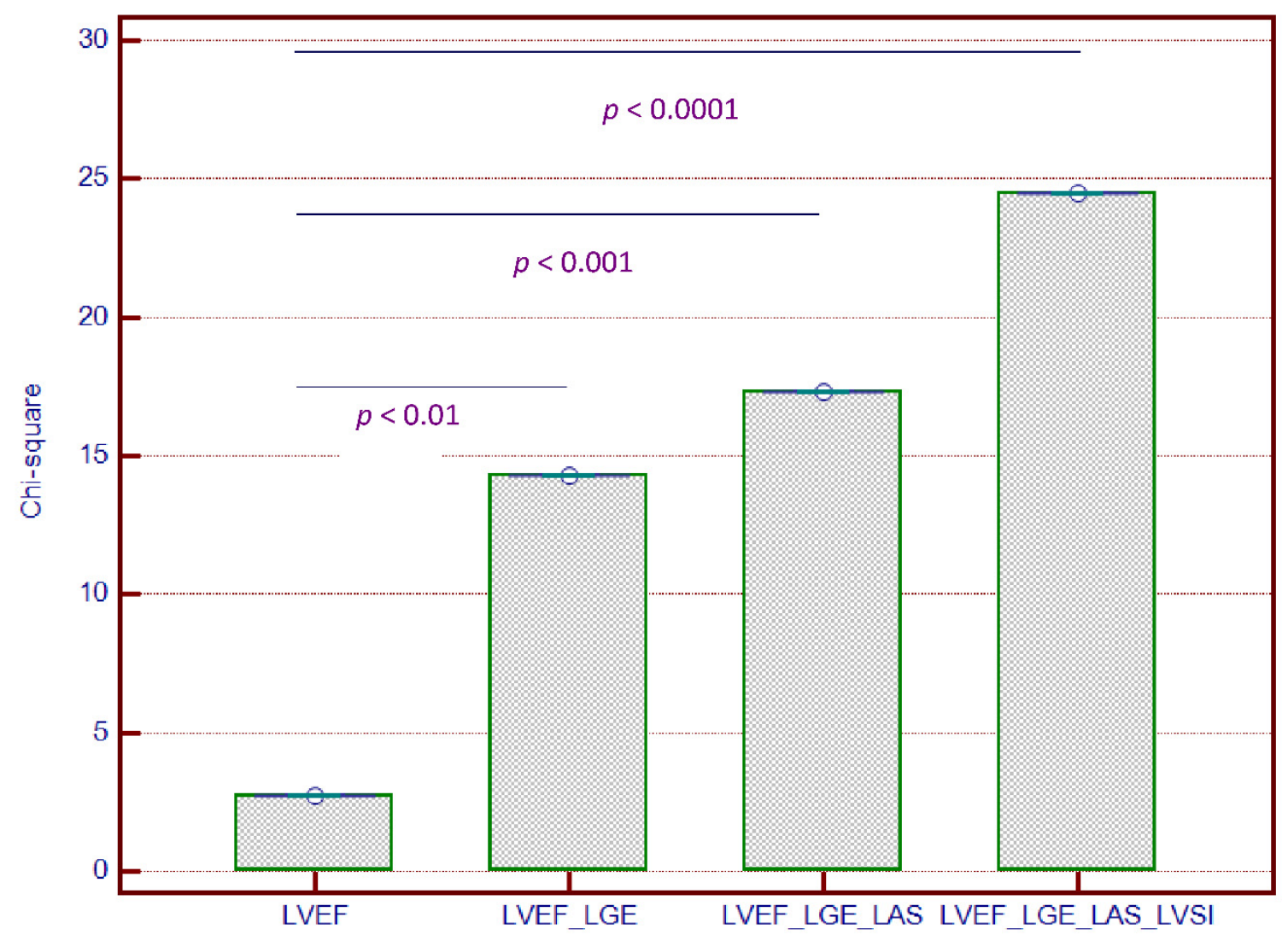

Figure 4. Incremental predictive value of LVSI and LAS added to LVEF and to LGE for outcome in patients with NIDCM. Abbreviations: LAS, left ventricular long axis strain; LGE, left ventricular late gadolinium enhancement; LVSI, left ventricular sphericity index; NIDCM, nonischemic dilated cardiomyopathy.

\subsection{Risk Stratification Scoring System}

The embedment of LVSI and LAS to LVEF and LGE allowed us to create a risk stratification score, using the following criteria: LVEF $<30 \%$, LGE+, LVSI $>0.48$ and LAS $<-7.8 \%$. These cut-off values were best correlated with outcome in our studied group. We created a scoring system and Kaplan-Meier curves based on the four parameters (Chi-square $=56.53, p<0.0001$ ) (Figure 5). We observed that patients with 3-4 points had significantly higher rates of MACEs during the follow-up period than others. 


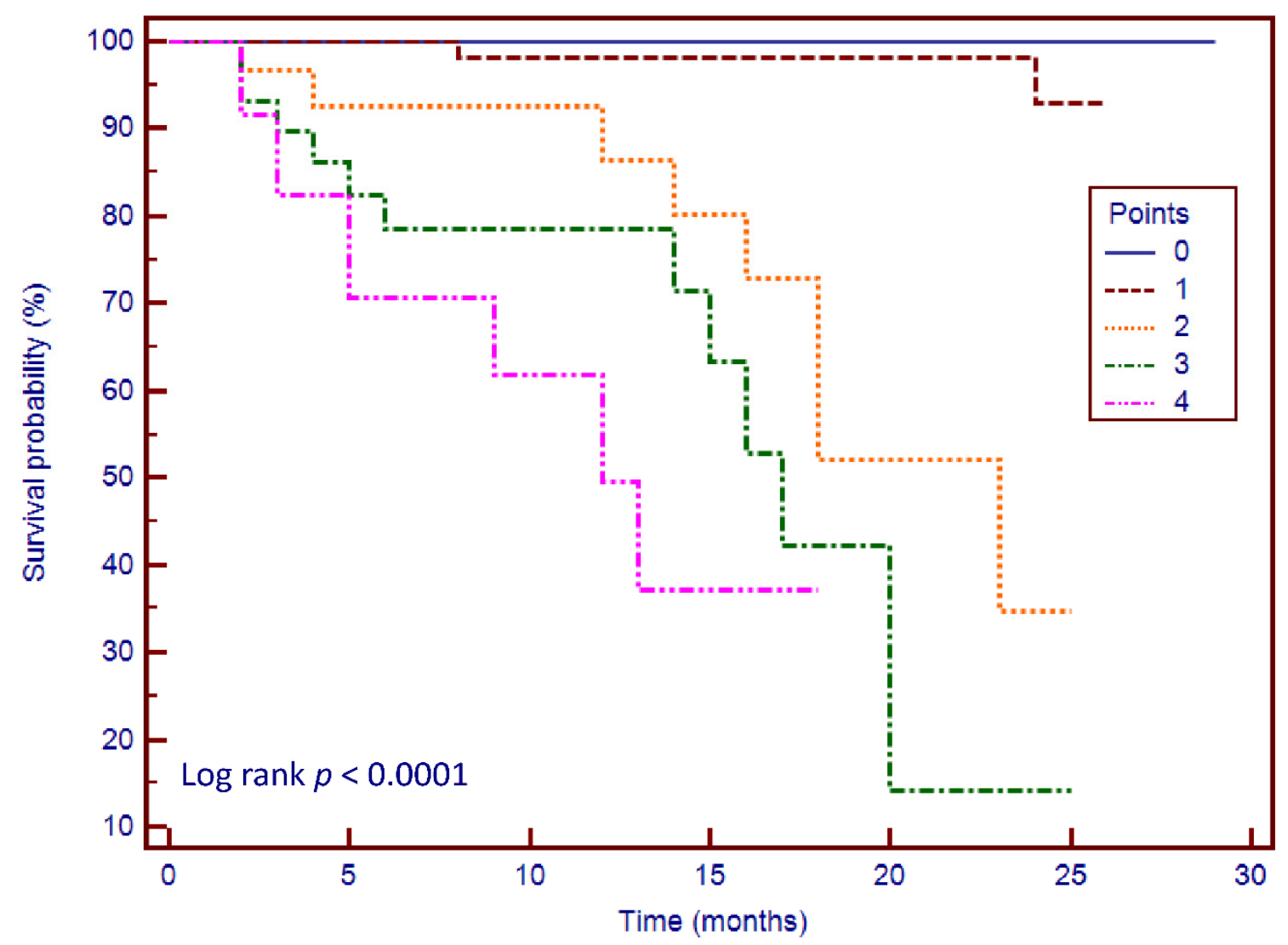

Figure 5. Kaplan-Meier curves for the risk stratification score. The scoring system ranges from 0 to 4 points: 1 point for each of the following LVEF $<30 \%$, LGE+, LAS $>-7.8 \%$ and LVSI $>0.48$. Abbreviations: LAS, left ventricular long axis strain; LGE, left ventricular late gadolinium enhancement; LVSI, left ventricular sphericity index.

\section{Discussion}

This prospective study is the first to evaluate the association between cMRI-based LV geometry and strain and outcome, in a significant, well-diagnosed NIDCM cohort. LAS and LVSI were independent predictors of MACEs in patients with NIDCM and myocardial replacement fibrosis. These findings were independent of LVEF and other established prognostic factors in a multivariable analysis. We also demonstrated that the addition of both LAS and LVSI to LVEF and LGE was superior for the prediction of MACEs over those based only on LVEF and LGE. The incidence of MACEs was higher in those with myocardial replacement fibrosis and altered LV geometry and strain, therefore representing a group who may require more aggressive therapy and rigorous follow-up.

NIDCM is typically associated with LV mid-wall replacement fibrosis, which worsens its prognosis [21,22]. Furthermore, Lehrke et al. have shown that NIDCM can be confirmed by cMRI-determined LV midwall fibrosis [23]. In our study, the incidence of LGE in NIDCM was similar with other studies $[8,24]$ and, in addition, we demonstrated that LGE was an independent predictor of MACEs. Similarly, in patients with NIDCM, Gulati et al. showed that the presence of mid-wall LGE was an independent predictor for outcome and improved risk stratification beyond LVEF [9]. Furthermore, several studies have confirmed that the presence LGE is independently associated with all-cause mortality, SCD and aborted SCD $[25,26]$.

In our study, we confirmed the role of LVSI in predicting MACEs with a cut-off value of $>0.43(p<0.001)$, similar with other published data. In patients with NIDCM, LVSI was initially evaluated in 2- and 3-dimensional echocardiography-based studies, which demonstrated that it was an independent predictor of MACEs, having a significant long-term prognostic impact [15,27]. 
Furthermore, in cMRI-based studies, it has been confirmed that LVSI is inversely correlated with LVEF in patients with NIDCM $[28,29]$, while in a multi-ethnic study conducted on healthy subjects, lowest LVSI was an independent predictor for CHD, CVD and HF at 10-year follow-up and the highest LVSI was correlated with increased incidence of HF and atrial fibrillation [30]. Moreover, in a small study, LVSI was an independent predictor for correct ICD therapy [31]. Thereby, LVSI could become an important prediction parameter in this category of patients.

Furthermore, we demonstrated that LAS, a myocardial strain parameter, was an independent predictor for outcome in patients with NIDCM. cMRI-based myocardial strain has proved its utility in early diagnosing and predicting various cardiac diseases. In patients with myocardial infarction, Gjesdal et al. showed that LAS was progressively reduced in larger mitral insufficiency and was associated with the infarction mass [32], while Schuster et al. identified that the assessment of LAS provided incremental prognostic value for cardiovascular risk [33]. In a multi-ethnic study, LAS was also associated with LVEF and MACEs [34]. In a study conducted on patients with aortic stenosis, our research team identified that LAS was an independent predictor of outcome and provided incremental value beyond LVEF and LGE [35]. Lastly, in patients with NIDCM, a single study identified that LAS was an independent predictor for SCD, aborted SCD, heart transplantation and HF hospitalization [36].

The role of LVEF and LGE as independent predictors of MACEs in patients with NIDCM has been confirmed by recently published data. To our knowledge, only two studies have approached our goals, namely Kano et al. identified that the addition of LVSI to LGE significantly increased prognosis of MACEs [37], while Riffel et al. demonstrated that the addition of LAS to LGE provides incremental value for outcome prediction in patients with NIDCM [36]. Our investigation is the first to demonstrate that the combined addition of both LVSI and LAS to LVEF and LGE significantly increased the predictive power of outcome, thus conferring an incremental predictive value.

Based on these four parameters, we were able to create a risk stratification scoring system. Hitherto, a single study created a similar scoring system based on LAS, LVEF and LGE+ which provided significant predictive value [36]. We demonstrated that the addition of one point for each of these parameters (LVEF $<30 \%$, LGE + , LAS $>-7.8 \%$ and LVSI $>0.48$ ) is highly correlated with MACEs. In patients without these risk features (score $=0$ ), no MACEs were observed during follow-up. Thereby, we propose a combined risk score consisting of LVEF, LGE, LVSI and LAS in order to improved risk stratification.

Study limitations: Firstly, we conducted a single centre study. Secondly, we were unable to acquire T1 mapping sequences, and therefore extracellular volume and diffuse myocardial fibrosis could not be quantified. Additionally, the follow-up was, relatively speaking, not very long.

\section{Conclusions}

cMRI parameters of geometry and longitudinal strain, namely LVSI and LAS, are independently associated with increased risk of MACEs in NIDCM patients with myocardial replacement fibrosis confirmed by cMRI-LGE. For the first time, we demonstrate that the combined usage of LVSI and LAS provide incremental value beyond the assessment of LVEF and LGE in outcome prediction. These findings have potential therapeutic implications regarding the management of patients with NIDCM.

Author Contributions: B.O.C.-M. and L.A.-C. conception and design of the study; L.A.C.-acquisition, analysis and interpretation of data; statistical methods and interpretation of results; project administration. A.Z., I.D.M., C.C., D.H., E.K., R.R., R.C., and M.F.-acquisition, analysis and interpretation of data; critical revision of the manuscript for intellectual content. B.O.C.-M., A.Z. and L.A.-C. drafting of the manuscript. All authors granted final approval of the manuscript submitted. All authors have read and agreed to the published version of the manuscript.

Funding: This research received no external funding.

Acknowledgments: This work was supported by internal institutional doctoral fellowship from the Iuliu Hatieganu University of Medicine and Pharmacy of Cluj-Napoca.

Conflicts of Interest: The authors declare no conflict of interest. 


\section{References}

1. Pinto, Y.M.; Elliott, P.M.; Arbustini, E.; Adler, Y.; Anastasakis, A.; Böhm, M.; Duboc, D.; Gimeno, J.; de Groote, P.; Imazio, M.; et al. Proposal for a Revised Definition of Dilated Cardiomyopathy, Hypokinetic Non-Dilated Cardiomyopathy, and Its Implications for Clinical Practice: A Position Statement of the ESC Working Group on Myocardial and Pericardial Diseases. Eur. Heart J. 2016, 37, 1850-1858. [CrossRef] [PubMed]

2. Felker, G.M.; Thompson, R.E.; Hare, J.M.; Hruban, R.H.; Clemetson, D.E.; Howard, D.L.; Baughman, K.L.; Kasper, E.K. Underlying Causes and Long-Term Survival in Patients with Initially Unexplained Cardiomyopathy. N. Engl. J. Med. 2000, 342, 1077-1084. [CrossRef] [PubMed]

3. Kadish, A.; Dyer, A.; Daubert, J.P.; Quigg, R.; Estes, N.A.M.; Anderson, K.P.; Calkins, H.; Hoch, D.; Goldberger, J.; Shalaby, A.; et al. Prophylactic Defibrillator Implantation in Patients with Nonischemic Dilated Cardiomyopathy. N. Engl. J. Med. 2004, 350, 2151-2158. [CrossRef] [PubMed]

4. Ponikowski, P.; Voors, A.A.; Anker, S.D.; Bueno, H.; Cleland, J.G.F.; Coats, A.J.S.; Falk, V.; González-Juanatey, J.R.; Harjola, V.-P.; Jankowska, E.A.; et al. 2016 ESC Guidelines for the Diagnosis and Treatment of Acute and Chronic Heart Failure. Eur. Heart J. 2016, 37, 2129-2200. [CrossRef]

5. Al-Khatib, S.M.; Stevenson, W.G.; Ackerman, M.J.; Bryant, W.J.; Callans, D.J.; Curtis, A.B.; Deal, B.J.; Dickfeld, T.; Field, M.E.; Fonarow, G.C.; et al. 2017 AHA/ACC/HRS Guideline for Management of Patients With Ventricular Arrhythmias and the Prevention of Sudden Cardiac Death. J. Am. Coll. Cardiol. 2018, 72, e91-e220. [CrossRef]

6. Buxton, A.E.; Ellison, K.E.; Lorvidhaya, P.; Ziv, O. Left Ventricular Ejection Fraction for Sudden Death Risk Stratification and Guiding Implantable Cardioverter-Defibrillators Implantation. J. Cardiovasc. Pharmacol. 2010, 55, 450-455.

7. Iles, L.M.; Ellims, A.H.; Llewellyn, H.; Hare, J.L.; Kaye, D.M.; McLean, C.A.; Taylor, A.J. Histological Validation of Cardiac Magnetic Resonance Analysis of Regional and Diffuse Interstitial Myocardial Fibrosis. Eur. Heart J.Cardiovasc. Imaging 2015, 16, 14-22. [CrossRef]

8. Halliday, B.P.; Gulati, A.; Ali, A.; Guha, K.; Newsome, S.; Arzanauskaite, M.; Vassiliou, V.S.; Lota, A.; Izgi, C.; Tayal, U.; et al. Association Between Midwall Late Gadolinium Enhancement and Sudden Cardiac Death in Patients With Dilated Cardiomyopathy and Mild and Moderate Left Ventricular Systolic Dysfunction. Circulation 2017, 135, 2106-2115. [CrossRef]

9. Gulati, A.; Jabbour, A.; Ismail, T.F.; Guha, K.; Khwaja, J.; Raza, S.; Morarji, K.; Brown, T.D.H.; Ismail, N.A.; Dweck, M.R.; et al. Association of Fibrosis With Mortality and Sudden Cardiac Death in Patients with Nonischemic Dilated Cardiomyopathy. JAMA 2013, 309, 896. [CrossRef]

10. Di Marco, A.; Anguera, I.; Schmitt, M.; Klem, I.; Neilan, T.G.; White, J.A.; Sramko, M.; Masci, P.G.; Barison, A.; Mckenna, P.; et al. Late Gadolinium Enhancement and the Risk for Ventricular Arrhythmias or Sudden Death in Dilated Cardiomyopathy. JACC Heart Fail. 2017, 5, 28-38. [CrossRef]

11. Yancy, C.W.; Jessup, M.; Bozkurt, B.; Butler, J.; Casey, D.E.; Drazner, M.H.; Fonarow, G.C.; Geraci, S.A.; Horwich, T.; Januzzi, J.L.; et al. 2013 ACCF/AHA Guideline for the Management of Heart Failure. J. Am. Coll. Cardiol. 2013, 62, e147-e239. [CrossRef] [PubMed]

12. Coelho-Filho, O.R.; Mongeon, F.-P.; Mitchell, R.; Moreno, H.; Nadruz, W.; Kwong, R.; Jerosch-Herold, M. Role of Transcytolemmal Water-Exchange in Magnetic Resonance Measurements of Diffuse Myocardial Fibrosis in Hypertensive Heart Disease. Circ. Cardiovasc. Imaging 2013, 6, 134-141. [CrossRef] [PubMed]

13. Arenja, N.; Andre, F.; Riffel, J.H.; aus dem Siepen, F.; Hegenbart, U.; Schönland, S.; Kristen, A.V.; Katus, H.A.; Buss, S.J. Prognostic Value of Novel Imaging Parameters Derived from Standard Cardiovascular Magnetic Resonance in High Risk Patients with Systemic Light Chain Amyloidosis. J. Cardiovasc. Magn. Reson. 2019, 21, 53. [CrossRef]

14. Tsadok, Y.; Friedman, Z.; Haluska, B.A.; Hoffmann, R.; Adam, D. Myocardial Strain Assessment by Cine Cardiac Magnetic Resonance Imaging Using Non-Rigid Registration. Magn. Reson. Imaging 2016, 34, 381-390. [CrossRef] [PubMed]

15. Liang, Y.; Li, W.; Zeng, R.; Sun, J.; Wan, K.; Xu, Y.; Cao, Y.; Zhang, Q.; Han, Y.; Chen, Y. Left Ventricular Spherical Index Is an Independent Predictor for Clinical Outcomes in Patients With Nonischemic Dilated Cardiomyopathy. JACC Cardiovasc. Imaging 2019, 12, 1578-1580. [CrossRef] 
16. Muthalaly, R.G.; Kwong, R.Y.; John, R.M.; van der Geest, R.J.; Tao, Q.; Schaeffer, B.; Tanigawa, S.; Nakamura, T.; Kaneko, K.; Tedrow, U.B.; et al. Left Ventricular Entropy Is a Novel Predictor of Arrhythmic Events in Patients With Dilated Cardiomyopathy Receiving Defibrillators for Primary Prevention. JACC Cardiovasc. Imaging 2019, 12, 1177-1184. [CrossRef]

17. Kramer, C.M.; Barkhausen, J.; Flamm, S.D.; Kim, R.J.; Nagel, E. Standardized Cardiovascular Magnetic Resonance (CMR) Protocols 2013 Update. J. Cardiovasc. Magn. Reson. 2013, 15, 91. [CrossRef]

18. Cerqueira, M.D.; Weissman, N.J.; Dilsizian, V.; Jacobs, A.K.; Kaul, S.; Laskey, W.K.; Pennell, D.J.; Rumberger, J.A.; Ryan, T.; Verani, M.S. Standardized Myocardial Segmentation and Nomenclature for Tomographic Imaging of the Heart. Circulation 2002, 105, 539-542. [CrossRef]

19. Bondarenko, O.; Beek, A.; Hofman, M.; Kühl, H.; Twisk, J.; van Dockum, W.; Visser, C.; van Rossum, A. Standardizing the Definition of Hyperenhancement in the Quantitative Assessment of Infarct Size and Myocardial Viability Using Delayed Contrast-Enhanced CMR. J. Cardiovasc. Magn. Reson. 2005, 7, 481-485. [CrossRef]

20. Gao, P.; Yee, R.; Gula, L.; Krahn, A.D.; Skanes, A.; Leong-Sit, P.; Klein, G.J.; Stirrat, J.; Fine, N.; Pallaveshi, L.; et al. Prediction of Arrhythmic Events in Ischemic and Dilated Cardiomyopathy Patients Referred for Implantable Cardiac Defibrillator. Circ. Cardiovasc. Imaging 2012, 5, 448-456. [CrossRef]

21. Piek, A.; de Boer, R.A.; Silljé, H.H.W. The Fibrosis-Cell Death Axis in Heart Failure. Heart Fail. Rev. 2016, 21, 199-211. [CrossRef] [PubMed]

22. Doltra, A.; Amundsen, B.; Gebker, R.; Fleck, E.; Kelle, S. Emerging Concepts for Myocardial Late Gadolinium Enhancement MRI. Curr. Cardiol. Rev. 2013, 9, 185-190. [CrossRef] [PubMed]

23. Lehrke, S.; Lossnitzer, D.; Schob, M.; Steen, H.; Merten, C.; Kemmling, H.; Pribe, R.; Ehlermann, P.; Zugck, C.; Korosoglou, G.; et al. Use of Cardiovascular Magnetic Resonance for Risk Stratification in Chronic Heart Failure: Prognostic Value of Late Gadolinium Enhancement in Patients with Non-Ischaemic Dilated Cardiomyopathy. Heart 2011, 97, 727-732. [CrossRef]

24. Sree Raman, K.; Nucifora, G.; Leong, D.P.; Marx, C.; Shah, R.; Woodman, R.J.; Molaee, P.; Shirazi, M.G.; McGavigan, A.D.; De Pasquale, C.G.; et al. Long Term Prognostic Importance of Late Gadolinium Enhancement in First-Presentation Non-Ischaemic Dilated Cardiomyopathy. Int. J. Cardiol. 2019, 280, 124-129. [CrossRef] [PubMed]

25. Halliday, B.P.; Baksi, A.J.; Gulati, A.; Ali, A.; Newsome, S.; Izgi, C.; Arzanauskaite, M.; Lota, A.; Tayal, U.; Vassiliou, V.S.; et al. Outcome in Dilated Cardiomyopathy Related to the Extent, Location, and Pattern of Late Gadolinium Enhancement. JACC Cardiovasc. Imaging 2019, 12, 1645-1655. [CrossRef] [PubMed]

26. Becker, M.A.J.; Cornel, J.H.; van de Ven, P.M.; van Rossum, A.C.; Allaart, C.P.; Germans, T. The Prognostic Value of Late Gadolinium-Enhanced Cardiac Magnetic Resonance Imaging in Nonischemic Dilated Cardiomyopathy. JACC Cardiovasc. Imaging 2018, 11, 1274-1284. [CrossRef] [PubMed]

27. Stolfo, D.; Merlo, M.; Pinamonti, B.; Barbati, G.; Di Lenarda, A.; Sinagra, G. Evolution of Left Ventricular Sphericity Index in Idiopathic Dilated Cardiomyopathy: Clinical and Prognostic Implications. Eur. Heart J. 2013, 34 (Suppl. 1), P1196. [CrossRef]

28. Marchal, P.; Lairez, O.; Cognet, T.; Chabbert, V.; Barrier, P.; Berry, M.; Mejean, S.; Roncalli, J.; Rousseau, H.; Carrie, D.; et al. Relationship between Left Ventricular Sphericity and Trabeculation Indexes in Patients with Dilated Cardiomyopathy: A Cardiac Magnetic Resonance Study. Eur. Heart J.Cardiovasc. Imaging 2013, 14, 914-920. [CrossRef]

29. Ben Halima, A.; Zidi, A. The Cardiac Magnetic Resonance Sphericity Index in the Dilated Cardiomyopathy: New Diagnostic and Prognostic Marker. Arch. Cardiovasc. Dis. Suppl. 2018, 10, 42. [CrossRef]

30. Ambale-Venkatesh, B.; Yoneyama, K.; Sharma, R.K.; Ohyama, Y.; Wu, C.O.; Burke, G.L.; Shea, S.; Gomes, A.S.; Young, A.A.; Bluemke, D.A.; et al. Left Ventricular Shape Predicts Different Types of Cardiovascular Events in the General Population. Heart 2017, 103, 499-507. [CrossRef]

31. Nakamori, S.; Ismail, H.; Ngo, L.H.; Manning, W.J.; Nezafat, R. Left Ventricular Geometry Predicts Ventricular Tachyarrhythmia in Patients with Left Ventricular Systolic Dysfunction: A Comprehensive Cardiovascular Magnetic Resonance Study. J. Cardiovasc. Magn. Reson. 2017, 19, 79. [CrossRef] [PubMed]

32. Gjesdal, O.; Almeida, A.L.C.; Hopp, E.; Beitnes, J.O.; Lunde, K.; Smith, H.-J.; Lima, J.A.C.; Edvardsen, T. Long Axis Strain by MRI and Echocardiography in a Postmyocardial Infarct Population. J. Magn. Reson. Imaging 2014, 40, 1247-1251. [CrossRef] [PubMed] 
33. Schuster, A.; Backhaus, S.J.; Stiermaier, T.; Kowallick, J.T.; Stulle, A.; Koschalka, A.; Lotz, J.; Kutty, S.; Bigalke, B.; Gutberlet, M.; et al. Fast Manual Long-Axis Strain Assessment Provides Optimized Cardiovascular Event Prediction Following Myocardial Infarction. Eur. Heart J.Cardiovasc. Imaging 2019, 20, 1262-1270. [CrossRef] [PubMed]

34. Gjesdal, O.; Yoneyama, K.; Mewton, N.; Wu, C.; Gomes, A.S.; Hundley, G.; Prince, M.; Shea, S.; Liu, K.; Bluemke, D.A.; et al. Reduced Long Axis Strain Is Associated with Heart Failure and Cardiovascular Events in the Multi-Ethnic Study of Atherosclerosis. J. Magn. Reson. Imaging 2016, 44, 178-185. [CrossRef]

35. Agoston-Coldea, L.; Bheecarry, K.; Cionca, C.; Petra, C.; Strimbu, L.; Ober, C.; Lupu, S.; Fodor, D.; Mocan, T. Incremental Predictive Value of Longitudinal Axis Strain and Late Gadolinium Enhancement Using Standard CMR Imaging in Patients with Aortic Stenosis. J. Clin. Med. 2019, 8, 165. [CrossRef]

36. Riffel, J.H.; Keller, M.G.P.; Rost, F.; Arenja, N.; Andre, F.; aus dem Siepen, F.; Fritz, T.; Ehlermann, P.; Taeger, T.; Frankenstein, L.; et al. Left Ventricular Long Axis Strain: A New Prognosticator in Non-Ischemic Dilated Cardiomyopathy? J. Cardiovasc. Magn. Reson. 2016, 18, 36. [CrossRef]

37. Kano, N.; Okumura, T.; Hiraiwa, H.; Watanabe, N.; Kondo, T.; Fukaya, K.; Sawamura, A.; Morimoto, R.; Bando, Y.; Murohara, T. Prognostic Impact of Combination of Sphericity Index and Late Gadolinium Enhancement on Cardiac Magnetic Resonance in Patients with Dilated Cardiomyopathy. J. Card. Fail. 2016, 22, S177. [CrossRef]

(C) 2020 by the authors. Licensee MDPI, Basel, Switzerland. This article is an open access article distributed under the terms and conditions of the Creative Commons Attribution (CC BY) license (http://creativecommons.org/licenses/by/4.0/). 